\title{
Clinical value of combined detection of miR-1202 and miR-195 in early diagnosis of cervical cancer
}

\author{
XIELAN YANG ${ }^{1}$, ZHILING YAN $^{1}$, HONGYING YANG $^{1}$, HUIJING NI $^{1}$, LEI ZHANG $^{1}$ and YUFENG WANG ${ }^{2}$ \\ Departments of ${ }^{1}$ Gynecology and ${ }^{2}$ Oncology for the Elderly, The Third Affiliated Hospital of Kunming \\ Medical University and Yunnan Cancer Hospital, Kunming, Yunnan 650106, P.R. China
}

Received November 6, 2018; Accepted January 4, 2019

DOI: $10.3892 / \mathrm{ol} .2019 .9956$

\begin{abstract}
The clinical value of the combined detection of miR-1202 and miR-195 in the early diagnosis of cervical cancer was studied. A retrospective analysis of 70 cervical cancer patients treated in the The Third Affiliated Hospital of Kunming Medical University and Yunnan Cancer Hospital from October 2015 to December 2017 was performed, and the lesion tissues were used as the experimental group. Normal cervical tissues from another 67 healthy females confirmed by physical examination at the same period were selected as the control group. The FIGO staging criteria were used for staging of the cervical cancer patients, reverse transcription-quantitative polymerase chain reaction (RT-qPCR) method was used for the detection of the expression of miR-1202 and miR-195 in different tissues, and the receiver operating curve (ROC) was used for the analysis of the application values of miR-1202 and miR-195 diagnosis alone and their combined diagnosis in early cervical cancer patients. The levels of miR-1202 and miR-195 in the experimental group were lower than those in the control group $(\mathrm{P}<0.05)$. The differences were significant in the different stages of cervical cancer tissues $(\mathrm{P}<0.05)$. The later the staging of cervical cancer tissues were, the lower the levels of miR-1202 and miR-195 were. The sensitivities and area under the curve (AUC) values of miR-1202 and miR-195 in the combined diagnosis of early cervical cancer were significantly higher than those of miR-1202 and miR-195 alone. The expression levels of miR-1202 and miR-195 in the cervical cancer patients are different in different stages. Guiding clinical treatment and prognosis according to the results of combined detection is beneficial for the development of treatment for cervical cancer patients and for prognostic judgement, worthy of popularization and application.
\end{abstract}

Correspondence to: Dr Yufeng Wang, Department of Oncology for the Elderly, The Third Affiliated Hospital of Kunming Medical University and Yunnan Cancer Hospital, 519 Kunzhou Road, Kunming, Yunnan 650106, P.R. China

E-mail: f422p8@163.com; 13577037585@163.com

Key words: miR-1202, miR-195, cervical cancer, early diagnosis

\section{Introduction}

Cervical cancer has the second highest incidence of female common tumors, with the highest mortality rate among female cancers. Approximately 300,000 people die of it every year around the world (1). In recent years, the incidence of cervical cancer has increased and tends to be younger in age, posing a serious threat to the health of females (2). Without obvious symptoms in early onset, once found, cervical cancer is in the middle and late stage, with a poor prognosis. Therefore, its early diagnosis is of great significance for its treatment and prognosis (3).

At present, the pathogenesis of cervical cancer is still not fully clarified. Studies have shown that the occurrence and development of cervical cancer is an extremely complex multi-stage process regulated by many factors. The regulation of nucleic acid level is closely related to the occurrence of cervical cancer. In particular, the expression of target genes of non-coding microRNA (miRNA) is influenced by its target regulation, thereby affecting the occurrence and development of cervical cancer $(4,5)$. For example, miR-31 (6) has an impact on the biological function of cervical cancer cells by the target regulation of gene ARID1A.

In recent years, related studies have shown that miR-1202 can function in the occurrence and development of multiple tumors by inhibiting the proliferation of tumor cells. Among them, miR-1202 has been studied in breast cancer (7) and endometrial cancer (8). However, there is no related report on the expression and diagnostic value of it in cervical cancer. miR-195 is an important cancer suppressor gene (9). Studies have shown that it inhibits tumor cells in many tumors such as hepatocellular carcinoma (10), breast cancer (11) and non-small cell lung cancer (12). Yet, its expression and diagnostic value in cervical cancer is still not very clear.

Therefore, in this study, in order to provide a better plan for the early diagnosis of cervical cancer, the expression of miR-1202 and miR-195 in cervical cancer was explored. Thus, the clinical value of the combined detection of miR-1202 and miR-195 in the early diagnosis was investigated.

\section{Patients and methods}

General information. A retrospective analysis of 70 cervical cancer patients treated in The Third Affiliated Hospital of 
Kunming Medical University and Yunnan Cancer Hospital (Kunming, China) from October 2015 to December 2017 was performed, lesion tissues were used as the experimental group. The average age of the patients was $40.2 \pm 3.7$ years. According to the FIGO staging criteria, there were: stage I with 26 cases, stage II with 29 cases, stage III with 9 cases and stage IV with 6 cases. There were 47 cases of cervical squamous cell carcinoma and 23 cases of cervical adenocarcinoma according to the pathological type. Normal cervical tissues from another 67 healthy females confirmed by physical examination at the same period were selected as the control group. The average age was $41.1 \pm 2.8$ years. All the tissues were taken out and quickly stored at $-80^{\circ} \mathrm{C}$. There was no significant difference in age and BMI between the two groups of patients $(\mathrm{P}>0.05$; Table I).

Inclusion and exclusion criteria. Inclusion criteria were: i) the experimental group included patients confirmed with cervical cancer by pathology, and ii) the control group included females confirmed as healthy by physical examination. Exclusion criteria were: i) patients having undergone radiotherapy and chemotherapy prior to taking specimens; ii) patients with severe other organ diseases; iii) patients who did not cooperate with the examination. and iv) patients with cognitive and communication impairment. The participants and their family members signed an informed consent form to cooperate with the medical staff to complete relevant medical treatment and the study was approved by the Ethics Committee of The Third Affiliated Hospital of Kunming Medical University and Yunnan Cancer Hospital.

Experimental instruments and materials. Refrigerator at $-80^{\circ} \mathrm{C}$ (Sanyo, Tokyo, Japan); reverse transcription-quantitative polymerase chain reaction (RT-qPCR) (iQ5 Multicolor; Bio-Rad Laboratories, Inc., Hercules, CA, USA); TRIzol reagent (Invitrogen; Thermo Fisher Scientific, Inc., Waltham, MA, USA); RT-qPCR kit and minScript reverse transcription kit (Takara Biotechnology Co., Ltd., Dalian, China).

Methods. The lesion tissues and normal cervical tissues were taken out from the refrigerator at $-80^{\circ} \mathrm{C}$. Trizol reagent was added, tissue RNA was extracted, and $1 \mu 1$ of total RNA was obtained. The specific stem ring primers were added, respectively. Reverse transcription cDNA was performed according to the manufacturer's instructions. Reaction temperature: at $42^{\circ} \mathrm{C}$ for $15 \mathrm{~min}$ and at $85^{\circ} \mathrm{C}$ for 5 sec. Primers for miR-1202 and miR-195 were amplified according to sequence design, with U6 as an internal reference control (Table II). Amplification conditions: denaturation at $95^{\circ} \mathrm{C}$ for $30 \mathrm{sec}$, annealing at $60^{\circ} \mathrm{C}$ for $10 \mathrm{sec}$, extension at $72^{\circ} \mathrm{C}$ for $20 \mathrm{sec}$, for a total of 40 cycles, and extension at $72^{\circ} \mathrm{C}$ for $5 \mathrm{~min}$ to stop the reaction. The expression of miR-1202 and miR-195, resspectively, was calculated and analyzed using the $2^{-\Delta \mathrm{Cq}}$ method (13).

Statistical analysis. Chi-square test was used for enumeration data. Measurement data were expressed as mean \pm standard deviation, using the t-test. SPSS19.0 (Shanghai Yuchuang Network Technology Co., Ltd., Shanghai, China) statistical software was used for analysis. $\mathrm{P}<0.05$ was considered to indicate a statistically significant difference.

\section{Results}

Comparison of relative expression of miR-1202 and miR-195 between the two groups. The relative expression of miR-1202 in the experimental group was $0.45 \pm 0.13$, lower than that in the normal control group $0.97 \pm 0.32 ; \mathrm{P}<0.05$, and that of miR-195 in the experimental group was $0.53 \pm 0.15$, lower than that in the normal control group $0.96 \pm 0.25(\mathrm{P}<0.05$; Table III).

Comparison of relative expression of miR-1202 and miR-195 in different stages. In the cervical cancer patients of early stage (I-II) and middle-late stage (III-IV), the expression levels of miR-1202 in the experimental group were $0.65 \pm 0.22$ and $0.31 \pm 0.10$, respectively, and those of miR-195 were $0.78 \pm 0.18$ and $0.29 \pm 0.16$, respectively. Based on statistical analysis, there was a significant difference in the overall level $(\mathrm{P}<0.001)$, suggesting that the later the clinical staging is, the lower the expression levels of miR-1202 and miR-195 are (Table IV).

Diagnostic values of miR-1202 and miR-195 detection alone and their combined detection in early stage (I-II) of cervical cancer. Calculated with early patients and healthy population, in the diagnosis of early cervical cancer patients, the diagnosis standard of miR-1202 alone was $<0.845$. The sensitivity, specificity and area under the curve (AUC) value was $81.82 \%, 65.67 \%$ and 0.7225 , respectively. That of miR-195 diagnosis alone was $<0.950$. The sensitivity, specificity and AUC value was $80.00 \%$, $49.25 \%$ and 0.6544 , respectively. The combined diagnosis of miR-1202 and miR-195 was $89.09 \%, 58.21 \%$ and 0.7544 , respectively. Among them, the sensitivity and AUC value of the combined diagnosis were significantly higher than those of miR-1202 and miR-195 diagnosis alone (Table V and Fig. 1).

\section{Discussion}

Cervical cancer is one of the most common malignant tumors of the reproductive system in females, with the highest incidence that is still rising (14). Studies have reported (15) that early cervical cancer is not easy to be detected, but once found, it is often in the middle and late stage, with a higher recurrence, invasion and metastasis rate, leading to poor treatment and prognosis of cervical cancer patients. The pathogenesis of cervical cancer is a very complicated process, involving the abnormalities of the structures and expression of many coding and non-coding genes, from normal cervical epithelial cells to infiltration of cancer cells (16). As a single-stranded non-coding regulatory RNA, by participating in the regulation of target gene expression, miRNA plays a role in various biological processes such as cell proliferation, apoptosis, differentiation and migration (17). The biological function of miR-1202 is mainly related to cell proliferation and apoptosis. Studies of Du et al (18) showed that miR-1202 induced endoplasmic reticulum stress and apoptosis through the target regulation of Rab1A in glioma. It also inhibited the proliferation of glioma cells. Studies have shown (19) that as a cancer suppressor gene, miR-195 is closely related to the occurrence of breast cancer, bladder cancer, colorectal cancer and other tumors, through the regulation of target genes. In addition, related studies have reported that the expression of miR-195 is downregulated in the lesion tissues of cervical cancer. The downregulation is 
Table I. Comparison of general information between the two groups of patients [n (\%)].

\begin{tabular}{|c|c|c|c|c|}
\hline Factors & Experimental group $(n=70)$ & Control group $(n=67)$ & $\chi^{2}$ & P-value \\
\hline Age & & & 0.002 & 0.966 \\
\hline$\leq 40$ & $41(58.57)$ & $39(58.21)$ & & \\
\hline$>40$ & $29(41.43)$ & $28(41.79)$ & & \\
\hline BMI & & & 0.005 & 0.942 \\
\hline$\leq 22$ & $37(52.86)$ & $35(52.24)$ & & \\
\hline$>22$ & $33(47.14)$ & $32(47.76)$ & & \\
\hline Married or not & & & 0.055 & 0.814 \\
\hline Yes & $51(72.86)$ & $50(74.63)$ & & \\
\hline No & $19(27.14)$ & $17(25.37)$ & & \\
\hline Fertile or not & & & 0.165 & 0.685 \\
\hline Yes & $49(70.00)$ & $49(73.13)$ & & \\
\hline No & $21(30.00)$ & $18(26.87)$ & & \\
\hline Family history & & & - & - \\
\hline Yes & $23(32.86)$ & - & & \\
\hline No & $47(67.14)$ & - & & \\
\hline Staging & & & - & - \\
\hline Stage I & $26(37.14)$ & - & & \\
\hline Stage II & $29(41.43)$ & - & & \\
\hline Stage III & $9(12.86)$ & - & & \\
\hline Stage IV & $6(8.57)$ & - & & \\
\hline Pathological types & & & - & - \\
\hline Cervical squamous cell carcinoma & $47(67.14)$ & - & & \\
\hline Cervical adenocarcinoma & $23(32.86)$ & - & & \\
\hline
\end{tabular}

Table II. RT-qPCR reaction miRNA-related primers.

\begin{tabular}{lll}
\hline Factors & Upstream primer sequences & Downstream primer sequences \\
\hline miR-1202 & 5'-ATCCAGTGCGTGTCGTG-3' & 5'-TGCTGTGCCAGCTGCAGT-3' \\
miR-195 & 5'-TAGCAGCA-CAGAAATATTGGC-3' & 5'-TGCTGTGCCAGCTGCAGT-3' \\
U6 & 5'-GCTTCGGCAGCACATATACTA-AAAT-3' & 5'-CGCTTCACGAATTTGCGTGTCAT-3' \\
\hline
\end{tabular}

Table III. Comparison of relative expression of miR-1202 and miR-195 in the two groups.

\begin{tabular}{lcrr}
\hline Factors & Experimental group $(\mathrm{n}=70)$ & Control group $(\mathrm{n}=67)$ & $\mathrm{t}$ \\
\hline miR-1202 & $0.45 \pm 0.13$ & $0.97 \pm 0.32$ & 12.56 \\
miR-195 & $0.53 \pm 0.15$ & $0.96 \pm 0.25$ & 12.27 \\
\hline
\end{tabular}

Table IV. Comparison of relative expression of miR-1202 and miR-195 in different stages.

\begin{tabular}{lcc}
\hline Staging & miR-1202 & miR-195 \\
\hline Stage I-II $(\mathrm{n}=55)$ & $0.65 \pm 0.22$ & $0.78 \pm 0.18$ \\
Stage III-IV $(\mathrm{n}=15)$ & $0.31 \pm 0.10$ & $0.29 \pm 0.16$ \\
$\mathrm{t}$ & 5.800 & 9.554 \\
P-value & $<0.001$ & $<0.001$ \\
\hline
\end{tabular}


Table V. Diagnostic values of miR-1202 and miR-195 detection alone and their combined detection in early cervical cancer.

\begin{tabular}{lccr}
\hline Diagnostic methods & Sensitivity (\%) & Specificity (\%) & AUC \\
\hline miR-1202 & 81.82 & 65.67 & 0.7225 \\
miR-195 & 80.00 & 49.25 & 0.6544 \\
miR-1202 combined with miR-195 & 89.09 & 58.21 & 0.7544 \\
\hline
\end{tabular}

AUC, area under the curve.

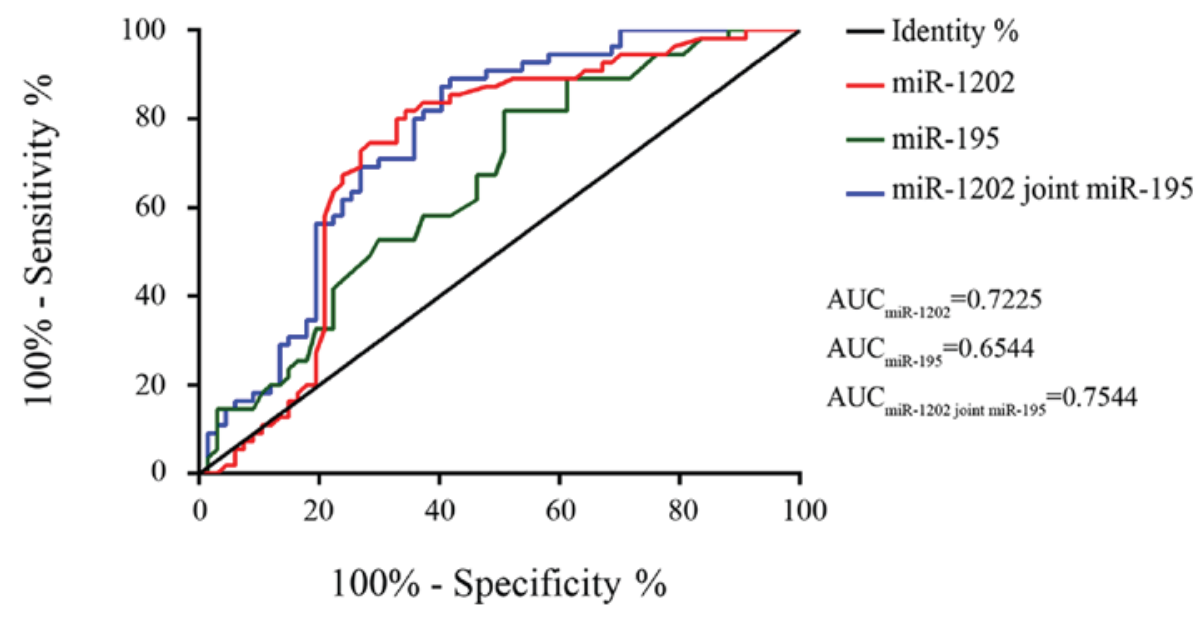

Figure 1. ROC curve of miR-1202 and miR-195 detection alone and their combined detection in early cervical cancer. Calculated with early patients and healthy population, in the diagnosis of early cervical cancer patients, the diagnosis standard of miR-1202 diagnosis alone was <0.845, and that of miR-195 diagnosis alone was $<0.950$. The AUC value of the ROC curve detected by miR-1202 alone was 0.7225 , and that detected by miR-195 alone was 0.6544 . That detected by miR-1202 combined with miR-195 was 0.7544 , significantly higher than that detected by miR-1202 and miR-195 alone. AUC, area under the curve; ROC, receiver operating curve.

associated with deep interstitial infiltration and lymph node metastasis in cervical cancer patients (20).

In order to clarify the diagnostic values of miR-1202 and miR-195 in cervical cancer, in this study, their expression in lesion and normal cervical tissues was compared. It was found that the expression in the experimental group was lower than that in the control group $(\mathrm{P}<0.05)$. Then, the relative expression of miR-1202 and miR-195 in different stages was compared. The results showed that the later the clinical staging was, the lower the expression levels were $(\mathrm{P}<0.05)$. The study of Wipperman et al (21) showed that miR-1202 played a role as a tumor suppressor gene in cervical cancer, so its expression in cervical cancer was downregulated. In the study of Philp et al (22), it was found that miR-195 could act as a cancer suppressor gene in cervical cancer, so its expression in cervical cancer tissues was downregulated compared to normal tissues. The results are consistent with our findings, confirming the role of miR-1202 and miR-195 as cancer suppressor genes in cervical cancer. The diagnostic values of miR-1202 and miR-195 detection alone and their combined detection in early cervical cancer were evaluated. The results showed that the sensitivity, specificity and AUC value of miR-1020 diagnosis alone was $81.82 \%, 65.67 \%$ and 0.7225 , respectively and in miR-195 diagnosis alone was $80.00 \%, 49.25 \%$ and 0.6544 , respectively, and in the combined diagnosis of miR-1202 and miR-195 was $89.09 \%, 58.21 \%$ and 0.7544 , respectively. The sensitivity and AUC value of the combined diagnosis were significantly higher than those of miR-1202 and miR-195 diagnosis alone, showing that the value of the combined diagnosis of miR-1202 and miR-195 in early cervical cancer is higher than that of their diagnosis alone.

In conclusion, the expression of miR-1202 and miR-195 in cervical cancer tissues is significantly downregulated. The value of the combined detection is higher than that of the detection alone in early cervical cancer, which can be used as a preferred solution for the clinical diagnosis of early cervical cancer. However, there are few research studies on miR-1202 and miR-195 in cervical cancer, and the sample size is small. Therefore, more investigations are required to carry out extensive research.

\section{Acknowledgements}

Not applicable.

\section{Funding}

This study was supported by Health Science and Technology Project of Yunnan Province (2017NS189).

\section{Availability of data and materials}

The datasets used and/or analyzed during the present study are available from the corresponding author on reasonable request. 


\section{Authors' contributions}

XY conceived the study and drafted the manuscript. HY directed the project and was also involved in the conception and design of the study. ZY, LZ and HN acquired the data. LZ and YW analyzed the data and revised the manuscript. All authors read and approved the final manuscript.

\section{Ethics approval and consent to participate}

The study was approved by the Ethics Committee of The Third Affiliated Hospital of Kunming Medical University and Yunnan Cancer Hospital (Kumming, China) and the participants and their family members signed an informed consent form.

\section{Patient consent for publication}

Not applicable.

\section{Competing interests}

The authors declare that they have no competing interests.

\section{References}

1. Turkistanli EC, Sogukpinar N, Saydam BK and Aydemir G: Cervical cancer prevention and early detection-the role of nurses and midwives. Asian Pac J Cancer Prev 4: 15-21, 2003.

2. Shi YR, Liu J, He W and Yang Y: Expression of Micro-RNA 218 in cervical cancer and its effect on proliferation, apoptosis and invasion of HeLa cells. Sichuan Da Xue Xue Bao Yi Xue Ban 47 697-702, 2016 (In Chinese).

3. Jung S, Yi L, Kim J, Jeong D, Oh T, Kim CH, Kim CJ, Shin J, An S and Lee MS: The role of vimentin as a methylation biomarker for early diagnosis of cervical cancer. Mol Cells 31: 405-411, 2011.

4. Shen Y, Chen H, Gao L, Zhang W, He J, Yang X, Qin L, Xue X and Guo Z: MiR-638 acts as a tumor suppressor gene in gastric cancer. Oncotarget 8: 108170-108180, 2017.

5. Zhang WN, Li W, Wang XL, Hu Z, Zhu D, Ding WC, Liu D, Li KZ, Ma D and Wang H: CLDN1 expression in cervical cancer cells is related to tumor invasion and metastasis. Oncotarget 7 : 87449-87461, 2016.

6. Yang X, Da M, Zhang W, Qi Q, Zhang C and Han S: Role of Lactobacillus in cervical cancer. Cancer Manag Res 10: 1219-1229, 2018

7. Chen H, Fan Y, Xu W, Chen J, Meng Y, Fang D and Wang J: Exploration of miR-1202 and miR-196a in human endometrial cancer based on high throughout gene screening analysis. Oncol Rep 37: 3493-3501, 2017.
8. McMullen JRW, Selleck M, Wall NR and Senthil M: Peritoneal carcinomatosis: limits of diagnosis and the case for liquid biopsy. Oncotarget 8: 43481-43490, 2017.

9. Zhou Q, Han LR, Zhou YX and Li Y: MiR-195 Suppresses cervical cancer migration and invasion through targeting Smad3. Int J Gynecol Cancer 26: 817-824, 2016.

10. Yan JJ, Chang Y, Zhang YN, Lin JS, He XX and Huang HJ: miR-195 inhibits cell proliferation via targeting AEG-1 in hepatocellular carcinoma. Oncol Lett 13: 3118-3126, 2017.

11. Nadeem F, Hanif M, Ahmed A, Jamal Q and Khan A: Clinicopathological features associated to MiRNA-195 expression in patients with breast cancer: evidence of a potential biomarker. Pak J Med Sci 33: 1242-1247, 2017.

12. Feng C, Zhang L, Sun Y, Li X, Zhan L, Lou Y, Wang Y, Liu L and Zhang Y: GDPD5, a target of miR-195-5p, is associated with metastasis and chemoresistance in colorectal cancer. Biomed Pharmacother 101: 945-952, 2018.

13. Livak KJ and Schmittgen TD: Analysis of relative gene expression data using real-time quantitative PCR and the 2(-Delta Delta C(T)) method. Methods 25: 402-408, 2001.

14. $\mathrm{Li} \mathrm{H}, \mathrm{Wu} \mathrm{X}$ and Cheng $\mathrm{X}$ : Advances in diagnosis and treatment of metastatic cervical cancer. J Gynecol Oncol 27: e43, 2016.

15. Zhou Z, Liu X, Hu K and Zhang F: The clinical value of PET and $\mathrm{PET} / \mathrm{CT}$ in the diagnosis and management of suspected cervical cancer recurrence. Nucl Med Commun 39: 97-102, 2018.

16. Lv KT, Liu Z, Feng J, Zhao W, Hao T, Ding WY, Chu JP and Gao LJ: MiR-22-3p regulates cell proliferation and inhibits cell apoptosis through targeting the eIF4EBP3 gene in human cervical squamous carcinoma cells. Int J Med Sci 15: 142-152, 2018.

17. George OL and Ness SA: Situational awareness: Regulation of the myb transcription factor in differentiation, the cell cycle and oncogenesis. Cancers (Basel) 6: 2049-2071, 2014.

18. Du B, Zhang P, Tan Z and Xu J: MiR-1202 suppresses hepatocellular carcinoma cells migration and invasion by targeting cyclin dependent kinase 14. Biomed Pharmacother 96: 1246-1252, 2017.

19. Li Z, Wang H, Wang Z and Cai H: MiR-195 inhibits the proliferation of human cervical cancer cells by directly targeting cyclin D1. Tumour Biol 37: 6457-6463, 2016.

20. Mou Z, Xu X, Dong M and Xu J: MicroRNA-148b acts as a tumor suppressor in cervical cancer by inducing G1/S-phase cell cycle arrest and apoptosis in a caspase-3-dependent manner. Med Sci Monit 22: 2809-2815, 2016.

21. Wipperman J, Neil T and Williams T: Cervical cancer: evaluation and management. Am Fam Physician 97: 449-454, 2018.

22. Philp L, Jembere N, Wang L, Gao J, Maguire B and Kupets R: Pap tests in the diagnosis of cervical cancer: help or hinder? Gynecol Oncol 150: 61-66, 2018. International (CC BY-NC-ND 4.0) License. 